\title{
Properties of Irradiated CdTe Detectors
}

\section{O. Korchak*}

E-mail: korchakefzu.cz

\section{Carna}

E-mail: carna@fzu.cz

\section{Havranek}

E-mail: havramefzu.cz

\section{Marcisovsky}

E-mail: marcisovefzu.cz

\section{Tomasek}

E-mail: tamaseklefzu.cz

\section{Vrba}

E-mail: vrbaefzu.cz

Institute of Physics of the Academy of Sciences of the Czech Republic, Na Slovance 1999/2, Praha 8, 18221

\begin{abstract}
Cadmium Telluride (CdTe) is a compound semiconductor with a large atomic number. It has a large photon absorption cross section in comparison with the silicon and germanium and due to it's large bandgap width it can be operated at room temperature. These properties predetermine CdTe to wide range of applications: from medical and industrial radiographic imaging to space sciences. In this paper, several characteristics of a commercially available CdTe sensors from two manufacturers were compared. The CdTe sensor samples were subject of measurement of their basic electronic characteristics at different temperatures ranging from -40 to $+40^{\circ} \mathrm{C}$. Additionally, the effects of ${ }^{60} \mathrm{Co}$ gamma iradiation up to a dose of $100 \mathrm{kGy}$ were studied at room temperature.
\end{abstract}

The 23rd International Workshop on Vertex Detectors,

15-19 September 2014

Macha Lake, The Czech Republic

${ }^{*}$ Speaker. 


\section{Introduction}

Cadmium Telluride $(\mathrm{CdTe})$ is due to a high atomic number $(\mathrm{Z}(\mathrm{Cd})=48$ and $\mathrm{Z}(\mathrm{Te})=52)$ a favorable material for the production of sensors of $X$-ray radiation. Furthermore, CdTe detectors can be operated at room temperature.

Due to its mechanical and physical properties the CdTe is widely used material. Therefore, in the recent years the CdTe sensors were subject of study of their basic characteristics, effects of different types of radiation (gamma, electrons, positrons, protons, neutrons) $[1,2,3,4,5,6]$ etc. However the manufacturing technologies of CdTe crystals are continuously improving, with results in larger detectors with more uniform response and better radiation hardness.

In this work, we present a study of the basic electrical characteristics of the currently (May 2014) commercially available CdTe sensors at a temperature range of $\langle-40,+40\rangle^{\circ} \mathrm{C}$ and the effects of gamma radiation at room temperature by the means of I-V measurement to assess their potencial perfomance in modern medical imaging.

\section{Characteristics of CdTe detectors}

CdTe detectors are generally fabricated by using metal-semiconductor-metal (MSM) structures. There are two main sensor types: Ohmic type and Schottky type.

These two types of sensors differ only by the contact electrodes. Ohmic contacts are formed by metals with a high work function, such as gold or platinum. Ohmic electrodes are placed on both sides of the Ohmic sensor type. Schottky sensor type uses an ohmic contact on one side of the sensor (cathode) and a blocking contact implemented with an In/Ti on the other side (anode) [7]. The platinum contact is fabricated by electroless plating, while the indium and titanium contact are formed by vacuum evaporation [7]. A structure of both types of sensors is schematically shown in Fig.1.

Both, Ohmic and Schottky type sensors have been studied in this work. One Ohmic type sensor (size: $4.83 \times 4.83 \times 1 \mathrm{~mm}^{3}$ ) was purchased from manufacturer \#1, and two samples, one Ohmic and one Schottky type sensors (size: $4 \times 4 \times 1 \mathrm{~mm}^{3}$ ), were purchased from
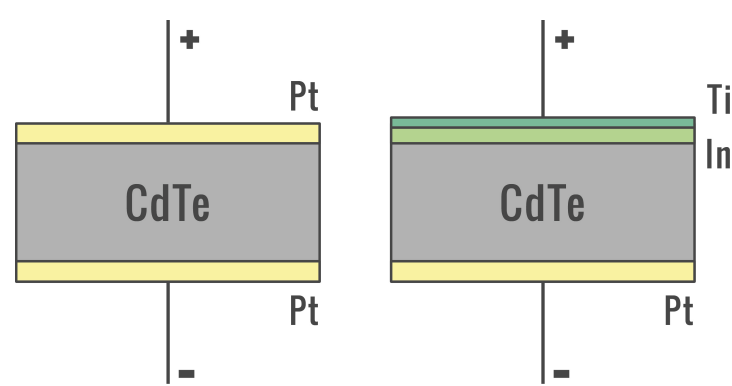

Figure 1: The structure of CdTe Ohmic (left) and Schottky (right) type detectors [7].

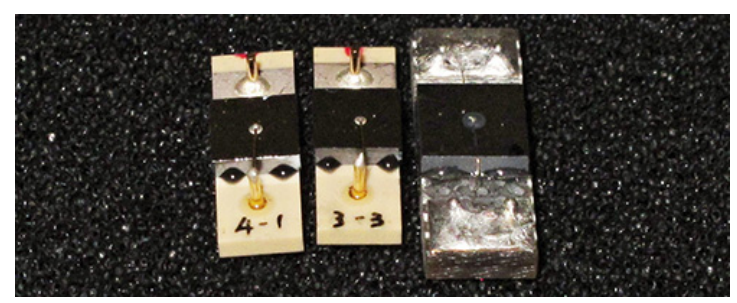

Figure 2: Tested samples: Schottky type sensor from manufacturer \#2 (left), Ohmic type sensors from manufacturer \#2 (center) and from manufacturer \#1 (right). manufacturer \#2.

Manufacturers recommend the optimum bias voltage to obtain the best energy spectrum. For the Ohmic type sensors it is $70 \mathrm{~V}$ and for the Schottky type sensors it is $700 \mathrm{~V}$. 
Fig.3 shows the I-V characteristics of the Ohmic (left) and the Schottky (right) sensors measured in a light-tight box at room temperature. The dark current of the Schottky type sensor is very small in comparison with the Ohmic type sensor. At $700 \mathrm{~V}$, it is only $10 \mathrm{nA}$, while for Ohmic type it is already $40 \mathrm{nA}$ at $70 \mathrm{~V}$. The difference of the leakage current between these sensor types is due to the fact that Schottky type sensors have a high resistance surface layer with almost constant negative charge density (the blocking layer or Schottky barrier) on the anode side. Therefore Schottky type sensors can be operated with a higher bias voltage of $700 \mathrm{~V}$, which means higher electric field and as a result, a higher charge collection destance. The Schottky type sensors biased with high voltage can potentially improve poor charge carriers transport properties typical for CdTe material due to the presence of defects and impurities in the crystals which act as trapping center [8].
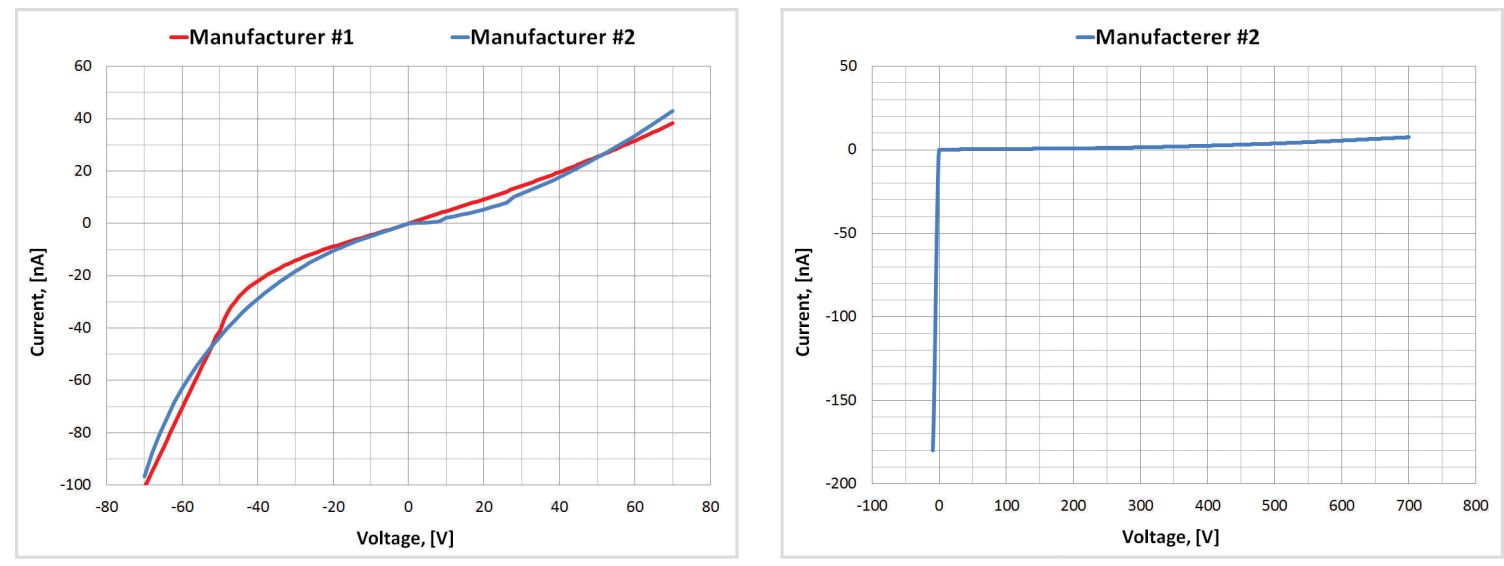

Figure 3: I-V curves of the CdTe Ohmic (left) and the Schottky (right) sensors.

Unlike the Si semiconductor detectors, where the capacitance depends on the depth of depleted zone, the capacitance of CdTe sensors is constant, because the active volume of the detector does not change with bias voltage. Fig.4 shows the $\mathrm{C}-\mathrm{V}$ curves measured in the light-tight box at room temperature with $1 \mathrm{MHz}$ frequency.
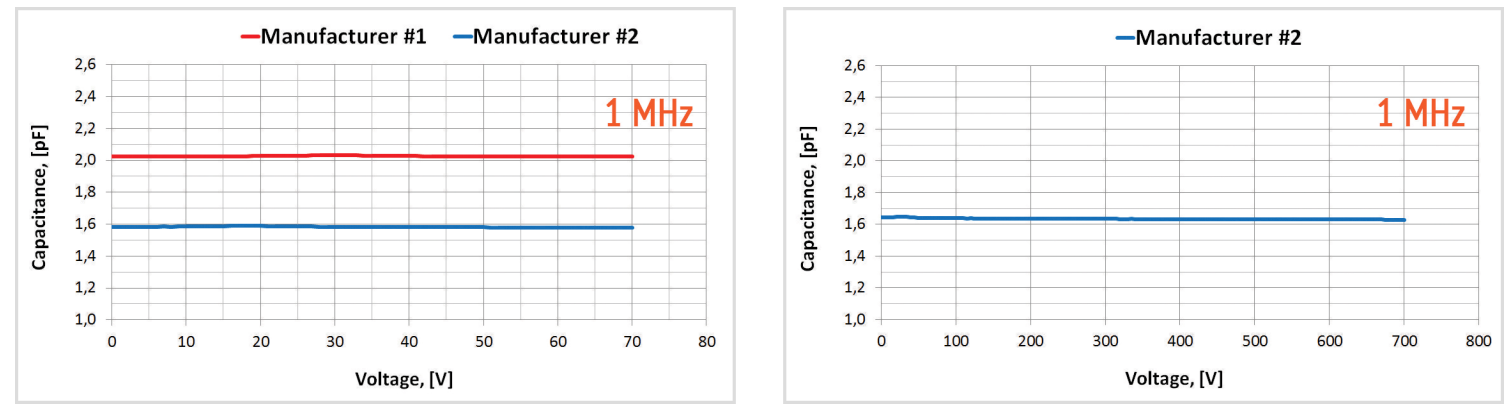

Figure 4: C-V curves of the CdTe Ohmic (left) and the Schottky (right) sensors.

\section{I-V measurements as a function of temperature}

The leakage current is a key parameter in characterising of spectrometric properties of the CdTe sensors, as the fluctuations of the leakage current are the most important source of noise 
in CdTe sensors [2]. Fig.5 shows leakage current of the Ohmic type (left) and the Schottky type (right) sensors measured in the temperature range of $\langle-40,40\rangle^{\circ} \mathrm{C}$. It can be seen that the leakage current of both sensor types decreases with the decrease of temperature. At the temperature of $40^{\circ} \mathrm{C}$, the dark current of the Ohmic type sensor at the bias voltage of $70 \mathrm{~V}$ increased from $43 \mathrm{nA}$ (at $20^{\circ} \mathrm{C}$ ) to $145 \mathrm{nA}$, and for the Schottky type sensor at the bias of $700 \mathrm{~V}$ from $10 \mathrm{nA}$ to $21 \mathrm{nA}$, but it still within acceptable range for spectrometric measurements. The values of the dark current of the Ohmic sensor type at $20^{\circ} \mathrm{C}(43 \mathrm{nA})$ and $0^{\circ} \mathrm{C}(6 \mathrm{nA})$ show, that cooling is a very effective way to decrease the leakage current and potentially improve spectrometry resolution of the CdTe sensors.
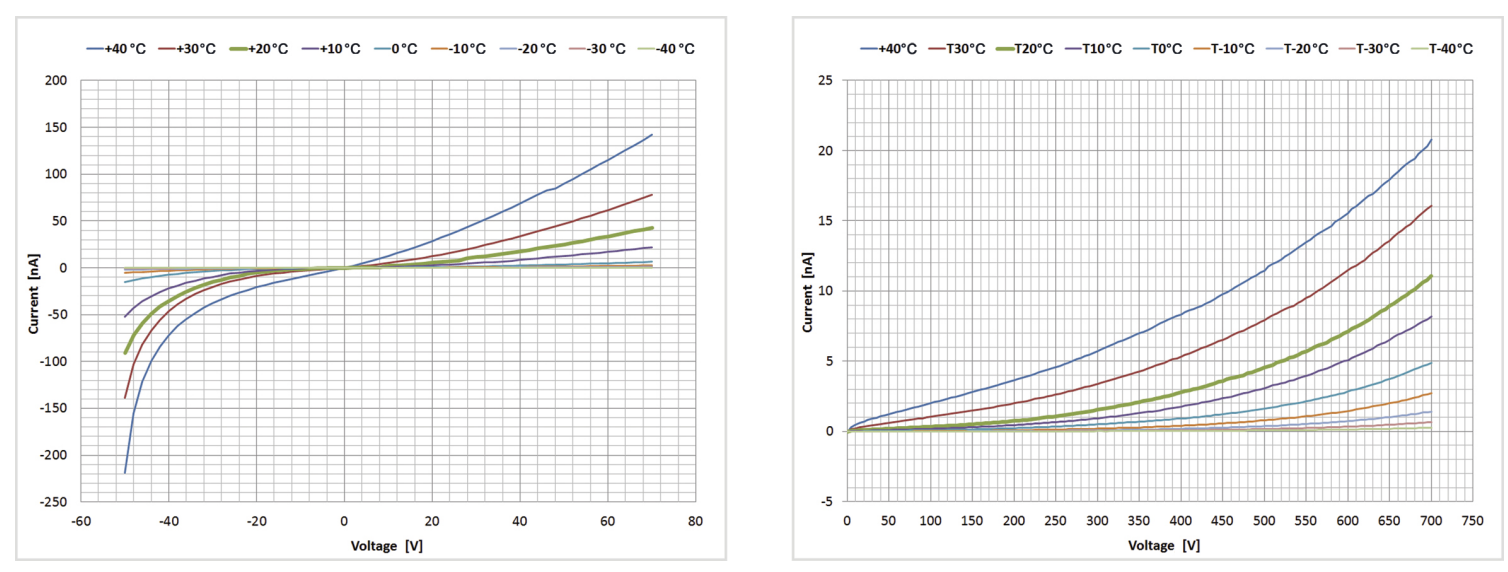

Figure 5: I-V curves of the CdTe Ohmic (left) and Schottky (right) sensors as a function of temperature. Manufacturer \#2.

\section{I-V measurements as a function of radiation dose}

The main application of the CdTe detectors is gamma rays detection. To assess the performance of these detectors under irradiation, both sensor types were exposed to radiation from a ${ }^{60} \mathrm{Co}$ source, which emits photons with the energy of $1.17 \mathrm{MeV}$ and $1.33 \mathrm{MeV}$. The total dose was $100 \mathrm{kGy}$ with a step of $10 \mathrm{kGy}$. The I-V characteristics were measured after each step at room temperature in the light-tight box. During irradiation, the temperature was kept constant at $24^{\circ} \mathrm{C}$. The time period between irradiation steps were about 30 minutes to make the I-V measurements.

Fig. 6 shows the I-V curves of Ohmic type sensors. After the first radiation step, $10 \mathrm{kGy}$, the dark current of sensors from both manufacturers decreased significatly from $43 \mathrm{nA}$ to $10 \mathrm{nA}$ at the bias voltage of $70 \mathrm{~V}$. Than up to dose of $50 \mathrm{kGy}$, resistance was kept roughly constant. For the absorbed dose in the range from $50 \mathrm{kGy}$ up to $100 \mathrm{kGy}$ the dark current increased to $50 \mathrm{nA}$ for positive bias voltage of $70 \mathrm{~V}$. For the negative bias voltage, both Ohmic type sensors had constant resistance from $10 \mathrm{kGy}$ up to $70 \mathrm{kGy}$. Then dark current of Ohmic sensor purchased from manufacturer \#1 for the absorbed dose from $70 \mathrm{kGy}$ to $100 \mathrm{kGy}$ increased from $10 \mathrm{nA}$ to $18 \mathrm{nA}$ at the bias voltage of $-70 \mathrm{~V}$, which is still lower than the dark current of an unirradiated sample. However, in the case of the Ohmic sensor from manufacturer \#2, the dark current after radiation dose of $90 \mathrm{kGy}$ at the bias of voltage of $-70 \mathrm{~V}$ was very large, and this sensor could not be operated with negative bias voltage of $-70 \mathrm{~V}$ after radiation dose $90 \mathrm{kGy}$. 

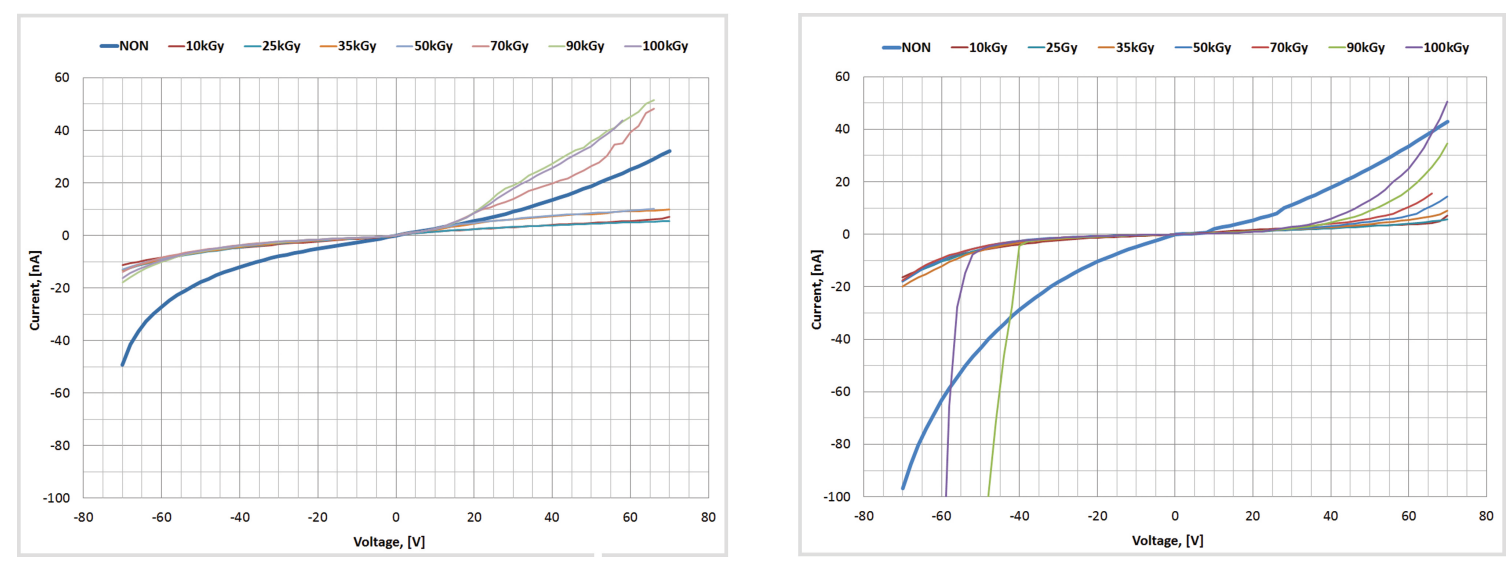

Figure 6: I-V curves of Ohmic type sensors from manufacturer \#1 (left) and manufacturer \#2 (right) as a function of radiation dose.

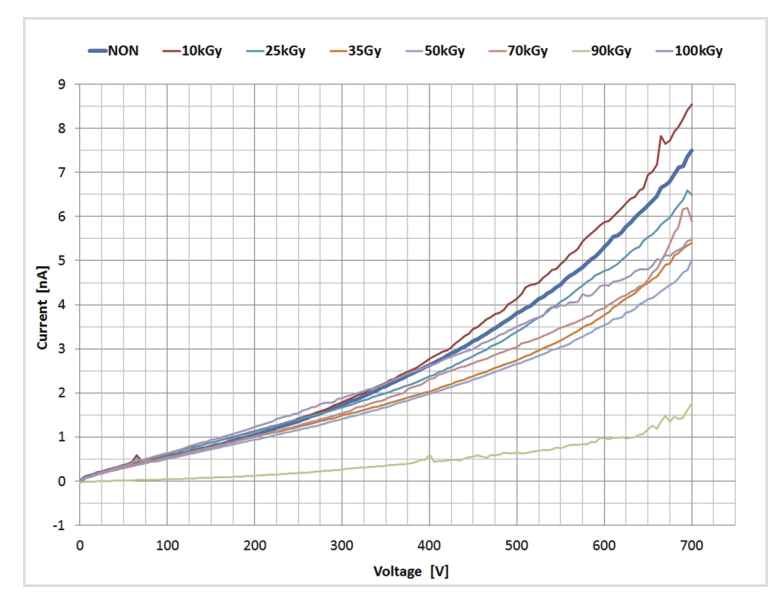

Figure 7: I-V curves of Schottky type sensor from manufacturer \#2 as a function of radiation dose.

As can be seen in Fig.7, resistance of the Schottky sensor type from manufacturer \#2 was not significantly affected by the ${ }^{60} \mathrm{Co}$ irradiation to the radiation dose of $100 \mathrm{kGy}$. The dark current was roughly constant during all measurements, it fluctuated from $2 \mathrm{nA}$ to $8 \mathrm{nA}$ at the bias voltage of $700 \mathrm{~V}$.

The results of leakage current measurements after gamma-ray irradiation differ from the results of the similar measurements since year 2000 [1], where after irradiation to the dose of $50 \mathrm{kGy}$ the leakage current of the sensor becomes almost 40 times bigger in comparison with the leakage current of the unirradiated sample at $70 \mathrm{~V}$, while in our case the leakage current after irradiation up to the dose of $50 \mathrm{kGy}$ is 3 times smaller in comparison with unirradiated sensor (Ohmic type sensor).

Moreover, the most interesting effect is that after low dose (10 kGy) of gamma-ray irradiation the leakage current decreased significatly for the Ohmic type sensors from both manufacturers, which can be explained by the reduction of the charge carrier lifetime of the bulk [1]. 


\section{Conclusions}

The basic characteristics of the currently (May 2014) commercially available CdTe sensors were investigated to assess their potential performance in modern medical imaging. The studied CdTe sensors were purchased from two different manufacturers. Two types of sensors differing by the contact electrodes have been tested: sensor with ohmic and with rectifying (Schottky) contacts.

The leakage current as most important source of noise in the CdTe sensors [2] was studied as a function of bias voltage in the temperature range of $\langle-40,40\rangle^{\circ} \mathrm{C}$. Additionally, I-V measurements after irradiation by ${ }^{60} \mathrm{Co}$ gamma-ray source to the radiation dose of $100 \mathrm{kGy}$ were performed at room temperature. Major conclusions of the measurements can be drawn as follows:

- Both sensor types from two manufacrurers show good performance in the temperature range of $\langle-40,40\rangle^{\circ} \mathrm{C}$. The leakage current is within acceptable range for spectrometric measurements under these conditions.

- After low dose (10 kGy) of gamma-ray irradiation the leakage current decreased significatly for Ohmic type sensors from both manufacturers, while leakage current of the Schottky type sensor was almost unaffected.

- Schottky type sensor show more stable response with increasing dose than the Ohmic type sensor in terms of leakage current.

- Leakage current of the CdTe sensors operated with positive biase voltage after irradiation by gamma-ray source ${ }^{60} \mathrm{Co}$ to the radiation dose of $100 \mathrm{kGy}$ increased for all tested samples but it is still within acceptable range for spectrometric measurements.

- The radiation hardness of the currently (May 2014) commercially available CdTe sensors is better in comparison with the sensors characterized with the similar measurements since year $2000[1]$ in terms of leakage current.

\section{Acknowledgement}

We acknowledge the support of the European Commission within the Framework Programme 7 Capacities, Grant Agreement 262025 and by the Ministry of Education, Youth and Sports of the Czech Republic under the project Nr. 7E12050.

\section{References}

[1] A. Cavallini, B. Fraboni and all, Electronic properties of traps induced by gamma-irradiation in CdTe and CdZnTe detectors, Nucl. Inst and Meth A 448 (2000) 558-566.

[2] A. Cavallini, M. Zanarini and all, Comparative evaluation of the temperature dependence of different noise sources in CdTe detectors, Nucl. Inst and Meth A 380 (1996) 127-131.

[3] A. Cavallini, B. Fraboni and all, Defetive states induced in CdTe and CdZnTe detectors by high and low energy neutron irradiation, Nucl. Inst and Meth B 213 (2004) 315-320. 
[4] M. Zanarini, P. Chirco and all, Radiation damage induced by $2 \mathrm{MeV}$ protons in CdTe and CdZnTe semiconductor detectors, Journal of Applied Physics, Vol. 94, Num. 5, 1 Sep 2003.

[5] A. Cavallini, B. Fraboni and all, Irradiation-induced defects in CdTe and CdZnTe detectors, Nucl. Inst and Meth A 458 (2001) 392-399.

[6] D. L. Baltzner, A. Romeo and all, High Energy Irradiation Properties Properties of CdTe/CdS Solar Cells, IEEE 2002, 0-7803-7471-1/02.

[7] Minoru Funaki, Development of CdTe detectors in Acrorad, Acrorad Co., Ltd Papers.

[8] Sensors 2009, 9, 3491-3526, [doi:10.3390/s90503491]

[9] A. Mohammadi, Measurement and Monte Carlo Calculation of the Response Function of a Schottky CdTe Detector with a Guard Ring Electrode for Medical X-ray Field, CYRIC AnnualReport, 2005.

[10] Tadayuki Takahashi, CdTe and CdZnTe Array Detectors, SNIC 2006. 